\title{
Positionierungen der Schulsozialarbeit in der französischsprachigen Schweiz
}

\author{
Hans-Peter von Aarburg und Jacques Kottelat
}

\section{Föderale Vielfalt}

Die Bildungslandschaft der Schweiz ist trotz breit abgestützter Harmonisierungsbemühungen föderal stark fragmentiert. Auch in den vier französischund den drei zweisprachigen Kantonen der Romandie arbeiten sonderpädagogische, schulpsychologische und sozialarbeiterische Dienste unter institutionell höchst unterschiedlichen Rahmenbedingungen.

Nicht nur unterscheiden sich institutionelle Gegebenheiten von einem welschen Kanton zum andern oft erheblich, auch der Kenntnisstand der im Bildungsbereich arbeitenden Fachpersonen über solche Gegebenheiten manchmal sogar anderer Kantonsteile - ist meist beschränkt. Nicht zuletzt auch die öffentliche Anerkennung der Schulsozialarbeit als einer Profession mit durchsetzbaren Standards leidet an einem dadurch behinderten fachlichen Austausch. Seit 2012 trifft sich deshalb eine Gruppe von französischsprachigen Schulsozialarbeitenden im Rahmen einer „Tour de Romandie“ einmal im $\mathrm{Jahr}$, um sich fachlich auszutauschen und dabei auch berufspolitisch zu positionieren. Ihren fünften Halt machte die Tour im Mai 2017 an der Fachhochschule für Soziale Arbeit in Sierre. Zusammen mit „AvenirSocial“, einem gesamtschweizerischen Verband von Professionellen der Sozialen Arbeit, organisierten die beiden Autoren dieses Treffen. An Schulen sozialarbeiterisch engagierte Fachpersonen aus allen ganz oder teilweise französischsprachigen Kantonen nahmen daran teil. ${ }^{1}$ Es wurde dabei die grosse institutionelle Vielfalt sichtbar, die im Folgenden skizziert werden soll. Mit Ausnahme des Unterwallis arbeiten heute Fachpersonen der Sozialen Arbeit in all diesen Kantonen an öffentlichen Schulen.

Jacques Kottelat (2015, S. 66f.), Koordinator der unter dem Dach von AvenirSocial organisierten Gruppe von welschen Schulsozialarbeitenden, führt in einer nach Kantonen geordneten Tabelle insgesamt 155 Stellen auf, die in der Romandie der Schulsozialarbeit (SSA) zugeordnet werden können.

1 Kurz zusammenfassende Dokumente zu diesem jährlichen Treffen finden sich unter: http://www.avenirsocial.ch/f/f42001506.html [Zugriff: 04.01.2017]. 
Dabei werden allerdings Stellen mit höchst unterschiedlichen Mandaten, organisatorischen Anbindungen und unterschiedlichen Bezeichnungen erfasst, was Vergleiche schwierig macht.

Diese föderale Vielfalt soll hier im Rahmen einer konzeptionellen Fragestellung umrissen werden. Empirisch beruht die Darstellung hauptsächlich auf persönlichen Recherchen über fachliche Netzwerke und auf öffentlich zugänglichen Dokumenten, wie sie sich etwa auf Internetportalen von Schulen, Gemeinden, Kantonen und Berufsverbänden finden. Daneben konnte gelegentlich auch auf Bachelor- und Masterarbeiten zurückgegriffen werden. Insgesamt ist dieser Fachbereich wenig dokumentiert.

Der vorliegende Beitrag möchte in einem Überblick Entwicklungslinien aufzeigen, er hat keinen Anspruch auf Vollständigkeit.

\section{1 „Schulinterne“ oder ,schulexterne“ Mandate?}

Fachpersonen der Sozialen Arbeit übernehmen auch in der Romandie an vielen Schulen sozialpädagogische, sozialarbeiterische und animatorische Mandate, die früher meist an Lehrpersonen übertragen waren. Die dazu notwendige Kooperation fordert alle Beteiligten heraus, Mandate zu verhandeln und sich zu positionieren: Wie viel Erziehung und Sozialarbeit hat die Schule neben der Wissensvermittlung und der Selektion zu übernehmen? In welchem Auftrag handelt die Soziale Arbeit?

Für alle direkt betroffenen Fachpersonen (und ihre Hierarchien) scheint es dabei wichtig zu sein, wieweit und in welchem Sinn sie Teil der Schule sind. Im Folgenden brauchen wir deshalb das Begriffspaar ,schulintern“ und "schulextern", um professionelle Mandate, wie wir sie beobachten, in ihrer Nähe bzw. Distanz zur Schule darzustellen. Diese Unterscheidung drängt sich auf, wie etwa aus Drillings (2009, S. 66) Darstellung von Kooperationsformen herausgeht, die auf Wilfried Wulfers und Mechthild Seithe beruht. Diese räumliche Kategorie verwenden auch die gemeinsam vom Schweizer Schularbeitsverband (ssav) und von AvenirSocial herausgegebenen Leitlinien (2010, 2016) explizit im Begriffspaar „intégré“/,,additionné“ (,integriert“/ „addiert"), auf die auch Kottelat (2015) zurückgreift.

Das Kriterium von ,innen“ und „aussen“ wird hier nacheinander unter vier verschiedenen Gesichtspunkten gesehen. Als Erstes wird die (Nicht-)Zugehörigkeit zu einer Profession der Schulpädagogik verstanden, als Zweites die organisatorische Einordnung der mit Mandaten Beauftragten in eine schulische bzw. eine sozialarbeiterische Hierarchie, als Drittes die Ausprägung einer partnerschaftlichen Kooperation mit den Lehrpersonen und als Viertes die räumliche Organisation des Mandates als eines „ambulanten“ bzw. „im 
Schulhaus integrierten" Dienstes. Um ein flüssiges Lesen zu ermöglichen, werden die einzelnen Beispiele jeweils möglichst zusammenhängend geschildert, auch wenn sie streng genommen aufgespalten und stets unter jedem der vier Punkte besprochen werden müssten.

\subsection{Sozial-oder schulpädagogische Mandate?}

Schule und Jugendhilfe in Genf spannten schon in den 1960er Jahren zusammen, um gemeinsam ein niederschwelliges Beratungsangebot im schulischen Alltag anzubieten (Dupuis \& Oldacre, 2013). Der Kanton finanziert seither an allen Schulen der Sekundarstufe 1 Fachpersonen der Sozialen Arbeit, die hauptberuflich Jugendliche, aber auch deren Eltern und Lehrpersonen beraten und unterstützend begleiten. Kottelat (2015) beziffert die Zahl dieser als "Sozialberaterinnen und -berater " („conseillers sociaux") bezeichneten Fachpersonen der Sozialen Arbeit mit 38. Rund ebenso viele Sozialberatende arbeiten auf der Sekundarstufe 2, also an verschiedenen Arten nachobligatorischer Ausbildungsgänge.

All diese Fachpersonen arbeiten in Schulgebäuden und sind ein integrierter Teil des pädagogisch tätigen Personals. Sie sind in einem eigenen Dienst der kantonalen Jugendhilfe organisiert. Über die Einzelfallarbeit hinaus wird von Sozialberaterinnen und -beratern insbesondere auch erwartet, dass sie sich für die Förderung eines vernetzenden Dialogs zwischen allen direkt oder indirekt im Schulbetrieb involvierten Akteurinnen und Akteuren einsetzen.

Als Sozialpädagoginnen und -pädagogen (,éducateurs sociaux") werden Fachpersonen der Sozialen Arbeit an Genfer Primarschulen vom Kanton seit 2006 beschäftigt. Sie arbeiten an Schulen, die als sozial besonders belastet eingestuft werden und deswegen im Rahmen des Programms REP (,réseau d'enseignement prioritaire") zusätzliche kantonale Mittel erhalten (Ferri, 2015). Die Sozialpädagoginnen und -pädagogen sollen in enger Zusammenarbeit insbesondere mit Lehrpersonen, der Schulleitung, dem schulpsychologischen Dienst und andern Institutionen die Integration der Schülerinnen und Schüler in den schulischen Alltag fördern. Ein wichtiger Teil des Mandates ist es, die Schule in ihrer Zusammenarbeit mit den Eltern zu unterstützen.

Ausgehend von der Waadt hat sich an den Schulen der Romandie (ausser in Genf) ein Modell für niederschwellige sozialpädagogische Unterstützung entwickelt, welches in der Deutschschweiz unbekannt ist. Lehrpersonen mit einer Zusatzausbildung setzen sich hier als „schulische Mediatorinnen und Mediatoren" für ein gutes Schulklima ein. Dazu unterstützen und beraten sie insbesondere Heranwachsende, zusätzlich aber auch Lehrpersonen und Eltern, vertraulich und auf freiwilliger Basis. „Schulische Mediation“ gehört in 
der ganzen Westschweiz (mit Ausnahme des Kantons Genf) zumindest an den Sekundarschulen meist schon seit Jahrzehnten zum niederschwelligen und vom regulären Schulbudget finanzierten Hilfsangebot. Das Zusatzmandat der Mediation wird den Lehrpersonen mit einer Unterrichtsentlastung abgegolten, im Kanton Fribourg etwa mit der Entlastung von einer Wochenlektion pro 120 Schülerinnen und Schüler, im Kanton Wallis mit einer solchen pro 250 Schülerinnen und Schüler. In einem Sammelband werden von Fachverantwortlichen aller Kantone der Romandie differenzierte Einblicke in kantonale Entwicklungen dieses Modells gegeben (Mirimanoff, 2013).

In den vielen Schulen der welschen Schweiz, wo Vertreterinnen und Vertreter der Schulpädagogik und der Sozialen Arbeit inzwischen alltäglich zusammenarbeiten, scheinen die spezifischen und komplementären Möglichkeiten der beiden Ansätze erkannt, die Aufgabenbereiche bei Überschneidungen aushandelbar zu sein. Im Kanton Fribourg etwa sind inzwischen „Schulmediation" und „Schulsozialarbeit" als komplementäre Angebote auf kantonaler Ebene geregelt. ${ }^{2}$

An allen Schulzentren des Kantons Waadt arbeiten vom Kanton finanzierte und organisierte Teams von Fachpersonen verschiedener Disziplinen, die sich um das physische, psychische und soziale Wohlergehen der Schülerinnen und Schüler bemühen. ${ }^{3} \mathrm{Zu}$ diesen Fachpersonen gehören neben Pflegefachleuten (,infirmières scolaires“) insbesondere auch die erwähnten „schulischen Mediatorinnen und Mediatoren“, also spezialisierte Fachpersonen der Schulpädagogik, bisher allerdings keine Fachpersonen der Sozialen Arbeit. Seit einem Wechsel an der Spitze des Erziehungsdepartementes scheinen mit dem Schuljahr 2017/2018 im Rahmen eines zweijährigen Pilotprojektes allerdings auch auf kantonaler Ebene Modelle aus dem Bereich der Sozialen Arbeit zum Zuge zu kommen, wie sie sich erfolgreich auf Gemeindeebene entwickelt haben ${ }^{4}$.

Wie verwirrend es sein kann, institutionelle Gegebenheiten über Sprachgrenzen hinweg zu vergleichen, zeigen etwa die von der Schweizerischen Konferenz der Erziehungsdirektoren zum Entwicklungsstand der „Schulsozialarbeit“" zusammengestellten Resultate einer Kurzbefragung kantonaler Dienststellen. ${ }^{5}$ Darin wird der Begriff, „Schulsozialarbeit" mit „service social scolaire“ übersetzt, worunter zwei professionelle Gruppen, die „conseillers

2 Reglement vom 19. April 2016 zum Gesetz über die obligatorische Schule (SchR), Artikel 19 https://bdlf.fr.ch/frontend/versions/4734?locale=de [Zugriff: 04.01.2018].

3 https://www.vd.ch/themes/formation/sante-a-lecole/equipes-psps/ [Zugriff: 04.01.2018].

4 Communiqué de presse de l'Etat de Vaud du 15 août 2017

https://www.bicweb.vd.ch/frame.aspx?pPage $=/$ communique.aspx?pObjectID $=636652[\mathrm{Zu}-$ griff: 04.01.2018].

5 http://www.edk.ch/dyn/16173.php [Zugriff : 26.08.2017]. 
sociaux“ (,Sozialberater") und die „médiateurs scolaires“ (,,schulischen Mediatoren"), als gleichwertig vorausgesetzt und erfasst werden.

\subsection{Organisatorisch der Schule oder der Jugendhilfe unterstellt?}

Wie in andern Kantonen gingen viele Initiativen, professionelle Jugendhilfe mit dem schulischen Alltag zu verbinden, auch im Kanton Waadt oft von Gemeinden (Meyer \& al., 2003) aus. Einzelne von diesen haben schon vor Jahrzehnten damit begonnen, Fachpersonen der Sozialen Arbeit damit zu betrauen, Schülerinnen und Schüler, Eltern und Lehrpersonen zu beraten und ihnen gegebenenfalls auch längerfristige, institutionell vernetzte Unterstützung anzubieten. Vielerorts werden diese Fachpersonen als „Beratende Schule-Elternhaus" („,onseillers école-famille“) bezeichnet. In der Stadt Lausanne, wo sie in einem eigenen, städtischen Sozialdienst organisiert sind, werden sie "Schulsozialassistentinnen/-assistenten" (,assistantes sociales scolaires") genannt. Die ersten von ihnen waren bereits in den frühen 1970er Jahren angestellt worden.

Gehen in der Waadt Initiativen für schulsozialarbeiterische Angebote offenbar eher von sozialen Diensten der Gemeinden als von Schulen aus, so scheinen sich entsprechende Angebote in den Kantonen Neuchâtel und Fribourg eher auf Anregung von Schulleitungen und in direkter Abhängigkeit von diesen zu entwickeln.

La Chaux-de-Fonds und Le Locle waren die ersten Gemeinden des Kantons Neuenburg, welche Fachpersonen der Sozialen Arbeit an ihren Schulen einstellten, dies ebenfalls schon in den 1970er Jahren. Inzwischen gibt es in allen sieben Schulkreisen des Kantons sozialpädagogische Dienste (,,services socio-éducatifs"). Je nach Schule werden diese an Primar- und Sekundarschulen tätigen Fachkräfte als „Sozialpädagogisch Beratende“ (,conseiller socio-éducatif") oder als "Schulassistentinnen und -assistenten“ (,assistants sociaux“) bezeichnet. Kottelat (2015) zählt 25 Personen, die unter einem dieser beiden Titel im Kanton Neuenburg beschäftigt sind. Diese Fachpersonen werden von den Gemeinden bezahlt, sind dabei aber direkt den Direktionen von Primar- und Sekundarschulen unterstellt. Ihr Mandat besteht darin, Schulleitungen, Lehrpersonen, Eltern und Schülerinnen und Schüler sowohl in akuten Krisen als auch in einem eher präventiven Sinn zu unterstützen und zu beraten. Aurélie Brunner (2014) zeichnet in ihrer Masterarbeit die Entwicklung dieser Institution in diesem Kanton nach. Im Kanton Neuenburg werden an einzelnen Schulen inzwischen zusätzlich auch Sozialpädagoginnen und -pädagogen beschäftigt, die nicht als Teil des sozialpädagogischen Dienstes gelten. Das Mandat dieser Fachpersonen der Sozialen Arbeit beruht 
dabei weniger als anderswo auf dem Prinzip der Freiwilligkeit: Sozialpädagoginnen und -pädagogen sollen Schulleitungen und Lehrpersonen gezielt von alltäglichen erzieherischen Aufgaben entlasten, etwa indem sie sozial auffällige und störende Schülerinnen und Schüler betreuen, wenn diese im Rahmen einer erzieherischen Sanktion für eine Lektion vom Unterricht ausgeschlossen werden.

Auch an den Schulen mehrerer Freiburger Gemeinden, so an den Sekundarschulen („Cycles d'orientation“) von Romont und Estavayer, werden Schulsozialarbeitende ("travailleurs sociaux scolaires") mit vergleichbaren Mandaten betraut. Diesen Fachpersonen, die einer Schulleitung unterstehen, ist es vorab aufgetragen, Schülerinnen und Schüler mit störendem Verhalten im Rahmen von erzieherischen Sanktionen zu betreuen. Ziel dabei ist es, problematische Entwicklungen möglichst frühzeitig mit einer massgeschneiderten Begleitung aufzufangen. Neben Einzelfall- und Gruppenarbeit sollen sich diese Fachpersonen auch in einem umfassenderen strukturpräventiven Sinn für ein gutes Schulklima einsetzen. Die Kosten für ihre Anstellung werden von Gemeinden und vom Kanton geteilt.

Die Schulsozialarbeit an den französischsprachigen Sekundarschulen der Stadt Freiburg hält unter ähnlichen institutionellen Rahmenbedingungen weit grössere Distanz zu den Schulleitungen und setzt stärker auf Unterstützung im Rahmen freiwilliger Beratung.

\subsection{Als aktive Partner in Schulteams eingebunden?}

Im ganzen Kanton Bern, auch im französischsprachigen Teil, gibt es institutionalisierte und gesetzlich geregelte Schulsozialarbeit, der Kanton beteiligt sich mit momentan 10 Prozent an ihren Kosten. So arbeiten in der zweisprachigen Stadt Biel inzwischen je vier deutsch- und französischsprachige Fachpersonen der Sozialen Arbeit. Ihre grosse Akzeptanz bei Lehrpersonen und Schulleitungen wurde erst kürzlich erneut bestätigt und hat zu einer deutlichen Erhöhung der Stellenprozente geführt. Die Sozialarbeitenden sind hier zuständig für alle Primar- und Sekundarschulen der Stadt, unterstellt sind sie einem städtischen Sozialdienst (Andreoli, 2016). Dabei sind sie - wie gewöhnlich auch andernorts - in schulische und ausserschulische Helfernetze eingebunden. Kottelat (2017) betont, wie wertvoll dieser interprofessionelle Austausch im Rahmen institutionalisierter regelmässiger Zusammenkünfte für den Schulbetrieb ist.

Nicht zuletzt wohl allseitig gute Erfahrungen mit der Bieler Schulsozialarbeit und auch ein kollegial engagierter Austausch zwischen Fachpersonen und Behörden dieser Stadt mit umliegenden französischsprachigen Gemein- 
den haben diese veranlasst, an ihren Primar- und Sekundarschulen ebenfalls Schulsozialarbeit einzuführen. Dies gilt für Moutier, Valbirse und Tramelan/Reconvilier. Weitere Gemeinden erwägen eine Einführung.

Schätzen Schulleitung und Lehrpersonen die Tätigkeit der hier eng in die Schule eingebundenen Fachpersonen der Sozialen Arbeit besonders deshalb, weil diese den Schulalltag mit einem im Vergleich zu den Lehrpersonen dezentriert schulexternen Blick sehen? Im Idealfall geben Lehrpersonen hier Erziehungsverantwortung nicht an Fachpersonen der Sozialen Arbeit ab, sondern teilen sie mit ihnen. Wenn über Einzelfälle hinaus eingespielte Verhaltensmuster reflexiv bearbeitet werden, bedeutet das letztlich Schulentwicklung. Eine solche kooperative Einbindung von Fachpersonen in Schulteams ist anspruchsvoll und wirft Fragen auf, die hier nur gestellt, nicht aber beantwortet werden können:

Wie weit können Fachpersonen der Sozialen Arbeit in ihrer Zusammenarbeit mit Schulen ihre professionelle Eigenständigkeit als aussenstehende Dritte bewahren? Wie weit gelingt es ihnen dabei, oft zu Konflikten führende Routinen im Schulalltag mit einem systemisch und sozialpolitisch geschärften Blick von aussen zu betrachten und auftretende Spannungen als Drittpersonen im Interesse aller Beteiligten zu entspannen? Können sie sich davor schützen, mit delegierten Hilfsfunktionen von der Schule vereinnahmt zu werden und dadurch ihre mediative Stellung zu verlieren?

\subsection{Niederschwellig in Schulgebäuden tätig?}

Neben sonderpädagogischen sind auch schulpsychologische Dienste in einzelnen Kantonen unterschiedlich eng in die Schulen eingebunden. In der Waadt etwa werden solche Dienste grundsätzlich in den Schulgebäuden angeboten und ermöglichen deshalb einen alltäglichen Austausch mit andern Akteuren des Schulbetriebs. Auch die hier im Auftrag von Gemeinden beschäftigten "Beraterinnen und Berater Schule Familie" arbeiten meist in Schulgebäuden.

Im Gegensatz dazu befanden sich etwa im Kanton Neuenburg bis vor kurzem schulnahe Dienste, insbesondere die Schulpsychologie, räumlich meist ausserhalb von Schulgebäuden. Räumliche Distanz bzw. Nähe dürfte alltägliche Praktiken stark beeinflussen.

Da sich Schulsozialarbeit als niederschwelliges Angebot versteht, sollten die Fachpersonen für die beratenen Personengruppen über möglichst kurze Wege erreichbar sein. Nun sind aber insbesondere Primarschulen oft über mehrere Standorte verteilt, was eine ständige Präsenz der SSA in vielen lokalen Gegebenheiten verunmöglicht. Kompromisse mit festgelegten Präsenz- 
zeiten in kleineren Schulhäusern oder der Möglichkeit, bei Bedarf eine Fachperson auch kurzfristig herbeizurufen, sind deshalb nötig. Die räumlich verstandenen Kategorien von "ambulanter" und ,integrierter" SSA (Iseli \& Grossenbacher-Wymann, 2013, S. 9) sollten deswegen in einem graduellen Sinn angewandt werden.

Im Kanton Jura etwa arbeitet gegenwärtig eine Fachperson der Sozialen Arbeit an den verschiedenen Primar- und Sekundarschulen der Stadt Delémont unterschiedlich intensiv ,integriert", eine andere Fachperson der Sozialen Arbeit steht allen andern Schulen im Kanton ,ambulant“ zu Verfügung.

\section{Der Prozess der professionellen Positionierung}

Wie in der Deutschschweiz scheint sich die SSA auch in der Romandie hauptsächlich „,von unten nach oben“ zu entwickeln. Ganz offensichtlich fördern die für die ganze Schweiz typischen föderalen Systeme vielfältige Formen der schulischen oder schulnahen Jugendhilfe auf Gemeindeebene, die sich erst allmählich in kantonalen und noch weiter gefassten Strukturen verfestigen. Die SSA der Westschweiz befindet sich in weiten Teilen noch in einer Pionierphase, in der einerseits vieles möglich ist, anderseits aber auch institutionelle Sicherheit und verlässliche Orientierungspunkte fehlen.

In der berufssoziologischen Tradition von Hughes und Abbot (2003) kann beobachtet werden, wie sich hier eine neue Berufsgruppe mit ihren Zielen und Methoden auf einem konkreten Feld gegenüber andern Akteuren behauptet. Diese Akteure sind Schulbehörden und Dienste der Jugendhilfe auf kantonaler und kommunaler Ebene, Schulleitungen, verschiedene Gruppen der Lehrerschaft. Sie gehen untereinander und mit Fachpersonen der Sozialen Arbeit Allianzen ein oder geraten in Rivalitäten. Dabei zeigen die Spannungsfelder je nach Kanton und zum Teil sogar nach Gemeinde oft ganz andere Muster und Eigendynamiken, die von aussen betrachtet zudem oft nur schwer nachvollziehbar sind.

Erstaunlicherweise beschäftigt sich in der ganzen Romandie momentan noch keine Fachhochschule eingehend mit der Thematik von Sozialer Arbeit an Schulen und stösst entsprechende Diskussionen an oder moderiert sie. Noch keine Fachhochschule bietet hier einen Weiterbildungskurs zu spezifischen Fragen in diesem Themenkreis an, etwa in Form eines CAS. Auseinandersetzungen mit dem Thema fanden bisher vor allem über Studienpraktiken und Bachelor- und Masterarbeiten statt.

Auch dass es in der Westschweiz einen Berufsverband für SSA wie erwähnt erst im Ansatz gibt, ist ein Hinweis darauf, dass die Pionierphase noch nicht abgeschlossen ist. Schulsozialarbeitende sind hier noch wenig überregi- 
onal organisiert, sie finden sich oft isoliert an ihren Arbeitsorten. Unter diesen Bedingungen berufliche Standards zu entwickeln und durchzusetzen, ist schwierig, zumal sich institutionelle Gegebenheiten wie erwähnt zumindest von Kanton zu Kanton meist stark unterscheiden und Modelle und Erfahrungen deshalb nicht ohne weiteres übertragbar sind. Ein überregionales berufspolitisches Engagement in der Freizeit bringt zwar nur selten einen unmittelbar sichtbaren Nutzen, ist aber unverzichtbar.

\section{Literatur}

Abbott, A. (2003). Ecologies liées. Apropos du système des professions. In P.-M. Menger (Ed.), Les professions et leurs sociologies (pp. 29-50). Paris: Editions de la Maison des sciences de l'homme.

Andreoli, L. (2016). Problématiques scolaires: un terrain de coopération pour les travailleurs sociaux et travailleuses sociales et les enseignant-e-s?. Sierre: Mémoire de bachelor: HETS.

AvenirSocial \& ssav. (2010). Lignes directrices: qualité dans le travail social en milieu scolaire.

AvenirSocial \& ssav. (2016). Leitbild Soziale Arbeit in der Schule - Lignes directrices du travail social en milieu scolaire. Luzern, Bern: https://ssav.ch/de/publikationen/grundlagenpapiere [Zugriff: 02.03.2018].

Brunner, A. (2014). Le travail social en milieu scolaire dans le canton de Neuchâtel. Travail de master HES-SO. Lausanne.

Drilling, M. (2009). Schulsozialarbeit. Antworten auf veränderte Lebenswelten (4. ed.). Bern: Haupt.

Dupuis, G. \& Oldacre, N. (2013). Etre travailleur social à l'école. La construction du rôle de conseiller social et ses enjeux dans un cycle d'orientation. Genève.

Ferri, A. (2015). Apporter un autre regard. En milieu scolaire primaire, la présence d'éducateurs est très récente. actualité sociale, 55(03), 9-10.

Iseli, D. \& Grossenbacher-Wymann, S. (2013). Travail social en milieu scolaire. Lignes directrices ( $3 \mathrm{e}$ édition ed.). Berne: DIP.

Kottelat, J. (2015). Tournée romande: les travailleurs sociaux sont de plus en plus nombreux en milieu scolaire. actualité sociale, 55(03), 7-9.

Kottelat, J. (2017). Le groupe social scolaire: une démarche qui tend à favoriser l'inclusion scolaire? In L. Gremion \& e. al. (Eds.), Vers une école inclusive. Regards croisés sur des défis actuels (pp. 39-54). Ottawa: Presses universitaires.

Meyer, V. et al. (2003). Schulsozialarbeit in der Romandie. In M. Drilling (Ed.), Schule und Soziale Arbeit. Aspekte interdisziplinärer Zusammenarbeit (S. 34-42). Basel: Verlag Fachhochschule für Soziale Arbeit beider Basel.

Mirimanoff, J. A. (Ed.) (2013). Médiation et jeunesse: mineurs et médiations familiales, scolaires et pénales en pays francophones. Bruxelles: Larcier. 